\title{
On Polynomials Solutions of Quadratic Diophantine Equations
}

\author{
Amara Chandoul \\ Institut Supérieure d'Informatique et de Multimedia de Sfax, Sfax, Tunisia \\ E-mail: amarachandoul@yahoo.fr \\ Received April 11, 2011; revised May 14, 2011; accepted May 25, 2011
}

\begin{abstract}
Let $P:=P(t)$ be a polynomial in $\mathbb{Z}[X] \backslash\{0,1\}$. In this paper, we consider the number of polynomial solutions of Diophantine equation $E: X^{2}-\left(P^{2}-P\right) Y^{2}-(4 P-2) X+\left(4 P^{2}-4 P\right) Y=0$. We also obtain some formulas and recurrence relations on the polynomial solution $\left(X_{n}, Y_{n}\right)$ of $E$.
\end{abstract}

Keywords: Polynomial Solutions, Pell’s Equation, Diophantine Equation

\section{Introduction}

A Diophantine equation is an indeterminate polynomial equation that allows the variables to be integers only. Diophantine problems have fewer equations than unknown variables and involve finding integers that work correctly for all equations. In more technical language, they define an algebraic curve, algebraic surface or more general object, and ask about the lattice points on it. The word Diophantine refers to the Hellenistic mathematician of the 3rd century, Diophantus of Alexandria, who made a study of such equations and was one of the first mathematicians to introduce symbolism into algebra. The mathematical study of Diophantine problems Diophantus initiated is now called Diophantine analysis. A linear Diophantine equation is an equation between two sums of monomials of degree zero or one. While individual equations present a kind of puzzle and have been considered throughout history, the formulation of general theories of Diophantine equations was an achievement of the twentieth century. For example, the equation $a x+b y=1$ is known the linear Diophantine equation. In general, the Diophantine equation is the equation given by

$$
a x^{2}+b x y+c y^{2}+d x+e y+f=0
$$

The equation $x^{2}-D y^{2}=N$, with given integers $D$ and $N$ and unknowns $x$ and $y$, is called Pell's equation If $D$ is negative, it can have only a finite number of solutions. If $D$ is a perfect square, say $D=a^{2}$, the equation reduces to $(x-a y)(x+a y)=N$ and again there is only a finite number of solutions. The most interesting case of the equation arises when $D \neq 1$ be a positive non-square.

Although J. Pell contributed very little to the analysis of the equation, it bears his name because of a mistake by Euler.

Pell's equation $x^{2}-D y^{2}=1$ was solved by Lagrange in terms of simple continued fractions. Lagrange was the first to prove that $x^{2}-D y^{2}=1$ has infinitly many solutions in integers if $D \neq 1$ is a fixed positive non-square integer. If the lenght of the periode of $\sqrt{D}$ is $l$, all positive solutions are given by $x=P_{2 v k-1}$ and $y=Q_{2 v k-1}$ if $k$ is odd, and by $x=P_{v k-1}$ and $y=Q_{v k-1}$ if $k$ is even, where $v=1,2, \cdots$ and $P_{n} / Q_{n}$ denotes the nth convergent of the continued fraction expansion of $\sqrt{D}$. Incidentally, $x=P_{(2 v-1)(k-1)}$ and $y=Q_{(2 v-1)(k-1)}$, $y_{v}: v=1,2, \cdots$, are the positive solutions of $x^{2}-D y^{2}=-1$ provided that $l$ is odd.

There is no solution of $x^{2}-D y^{2}= \pm 1$ other than $x_{v}, y_{v}: v=1,2, \cdots$ given by $\left(x_{1}+\sqrt{D} y_{1}\right)^{v}=x_{v}+\sqrt{D} y_{v}$, where $x_{1}, y_{1}$ is the least positive solution called the fundamental solution, which there are different method for finding it. The reader can find many references in the subject in the book [1].

We recall that there are many papers in which are considered different types of Pell's equation. Many authors such as Tekcan [2], Kaplan and Williams [3], Matthews [4], Mollin, Poorten and Williams [5], Stevenhagen [6] and the others consider eome specific Pell equations and their integer solutions. In [2,7], It 
considered the equation $x^{2}-D y^{2}= \pm 4$ and the equation $x^{2}-D y^{2}= \pm 9$, and he obtained some formulas for its integer solutions. He mentioned two conjecture which was proved by A. S. Shabani [8]. In [9], we extend the work in $[2,7]$ by considering the Pell equation $x^{2}-D y^{2}= \pm k^{2}$ when $D \neq 1$ be a positive non-square and $k \geq 2$, we obtain some formulas for its integer solutions.

In [10], A. Dubickas and J. Steuding are interested in the polynomial solutions of the Pell equation given by

$$
P^{2}-D Q^{2}=1
$$

where $D$ is a fixed polynomial, $P$ and $Q$ are polynomials in the same variables as $D$ and with coefficients in the same field or ring as those of $D$. The solutions $(P, Q)=(1,0)$ and $(1,0)$ are called trivial. All other solutions are called non-trivial. The main difficulty in solving polynomial Pell equations is to determine whether non-trivial solutions exist or not. In case, if there is at least one non-trivial solution, all solutions are obtained as powers of the smallest non-trivial solution. They prove this for polynomials $D(t)$ in one variable with coefficients in $\mathbb{C}$ The proof is purely algebraic and extends without change to arbitrary polynomials in several variables $D \in \mathbb{K}\left[t_{1}, \ldots, t_{\mathrm{s}}\right]$ over every field $\mathbb{K}$ of characteristic $\neq 2$.

In $[11,12]$, the number of integer solutions of Diophantine equation

$x^{2}-\left(t^{2}-t\right) y^{2}-(4 t-2) x+\left(4 t^{2}-4 t\right) y=0$ and Diophantine equation

$x^{2}-\left(t^{2}-t\right) y^{2}-(16 t-4) x+\left(16 t^{2}-16 t\right) y=0$ over $\mathbb{Z}$ is considered, where $t \geq 2$.

\section{Main Results}

Let $P:=P(t)$ be a polynomial in $\mathbb{Z}[X] \backslash\{0,1\}$. In this paper, we consider the number of polynomial solutions of Diophantine equation

$$
\begin{aligned}
E & : X^{2}-\left(P(t)^{2}-P(t)\right) Y^{2}-(4 P(t)-2) X \\
& +\left(4 P(t)^{2}-4 P(t)\right) Y=0
\end{aligned}
$$

We also obtain some formulas and recurrence relations on the polynomial solution $\left(X_{n}, Y_{n}\right)$ of $E$.

Note that the resolution of $E$ in its present form is difficult, that is, we can not determine how many solutions $E$ has and what they are. So, we have to transform $E$ into an appropriate Diophantine equation which can be easily solved. To get this let

$$
T:\left\{\begin{array}{l}
X=U+H \\
Y=V+K
\end{array}\right.
$$

be a translation for some $H$ and $K$.
By applying the transformation $T$ to $E$, we get

$$
\begin{aligned}
T(E) & :=\tilde{E}:(U+H)^{2}-\left(P(t)^{2}-P(t)\right)(V+K)^{2} \\
& -(4 P(t)-2)(U+H)+\left(4 P(t)^{2}-4 P(t)\right)(V+K) \\
& =0
\end{aligned}
$$

In (3), we obtain $U(2 H+2-4 P(t))$ and $V\left(-2 K P(t)^{2}+2 K P(t)+\left(4 P(t)^{2}-4 P(t)\right)\right)$ So we get $H=2 P(t)-1$ and $K=2$. Consequently for $X=U+2 P(t)-1$ and $Y=V+2$, we have the Diophantine equation

$$
\tilde{E}: U^{2}-\left(P(t)^{2}-P(t)\right) V^{2}=1
$$

which is a Pell equation.

Now, we try to find all polynomial solutions $\left(U_{n}, V_{n}\right)$ of $T(E)$ and then we can retransfer all results from $T(E)$ to $E$ by using the inverse of $T$.

Theorem 2.1: Let $\tilde{E}$ be the Diophantine equation in (3), then

1) The fundamental solution of $\tilde{E}$ is

$$
\left(U_{1}, V_{1}\right)=(2 P(t)-1,2)
$$

2) Define the sequence $\left(U_{n}, V_{n}\right)$ by

$$
\left\{\begin{array}{l}
\left(\begin{array}{l}
U_{1} \\
V_{1}
\end{array}\right)=\left(\begin{array}{l}
2 P(t)-1 \\
2
\end{array}\right) \\
\left(\begin{array}{l}
U_{n} \\
V_{n}
\end{array}\right)=\left(\begin{array}{cc}
2 P(t)-1 & 2 P(t)^{2}-2 P(t) \\
2 & 2 P(t)-1
\end{array}\right)^{n-1}\left(\begin{array}{l}
U_{1} \\
V_{1}
\end{array}\right), \forall n \geq 2
\end{array}\right.
$$

Then $\left(U_{n}, V_{n}\right)$ is a solution of $\tilde{E}$

3) The solutions $\left(U_{n}, V_{n}\right)$ satisfy the recurrence relations

$$
\left\{\begin{array}{l}
U_{n}=(2 P(t)-1) U_{n-1}+\left[2 P(t)^{2}-2 P(t)\right] V_{n-1} \\
V_{n}=2 U_{n-1}+(2 P(t)-1) V_{n-1}
\end{array}\right.
$$

for $n \geq 2$

4) The solutions $\left(U_{n}, V_{n}\right)$ satisfy the recurrence relations

$$
\left\{\begin{array}{l}
U_{n}=(4 P(t)-3)\left(U_{n-1}+U_{n-2}\right)-U_{n-3} \\
V_{n}=(4 P(t)-3)\left(V_{n-1}+V_{n-2}\right)-U_{n-3}
\end{array}\right.
$$

for $n \geq 4$

5) The $n$-th solution $\left(U_{n}, V_{n}\right)$ can be given by 


$$
\frac{U_{n}}{V_{n}}=[P(t)-1 ; \underbrace{2,2 P(t)-2, \cdots, 2,2 P(t)-2}_{n-1 \text { times }}, 2], \forall n \geq 1
$$

Proof.

1) It is easily seen that $\left(U_{1}, V_{1}\right)=(\underset{\tilde{E}}{2} P(t)-1,2)$ is the fundamental polynomial solution of $\tilde{E}$ since

$$
(2 P(t)-1)^{2}-\left(P(t)^{2}-P(t)\right) 2^{2}=1
$$

2) We prove it using the method of mathematical induction. Let $n=1$, by (5) we get

$\left(U_{1}, V_{1}\right)=(2 P(t)-1,2)$ which is the fundamental solution and so is a solution of $\tilde{E}$. Now, we assume that the Diophantine equation (4) is satisfied for $n$, that is

$\tilde{E}: U_{n}^{2}-\left(P(t)^{2}-P(t)\right) V_{n}^{2}=1$. We try to show that this equation is also satisfied for $n+1$. Applying (5), we find that

$$
\begin{aligned}
\left(\begin{array}{c}
U_{n+1} \\
V_{n+1}
\end{array}\right)= & \left(\begin{array}{cc}
2 P(t)-1 & 2 P(t)^{2}-2 P(t) \\
2 & 2 P(t)-1
\end{array}\right)^{n}\left(\begin{array}{l}
U_{1} \\
V_{1}
\end{array}\right) \\
= & \left(\begin{array}{cc}
2 P(t)-1 & 2 P(t)^{2}-2 P(t) \\
2 & 2 P(t)-1
\end{array}\right) \\
& \cdot\left(\begin{array}{cc}
2 P(t)-1 & 2 P(t)^{2}-2 P(t) \\
2 & 2 P(t)-1
\end{array}\right)^{n-1}\left(\begin{array}{l}
U_{1} \\
V_{1}
\end{array}\right) \\
= & \left(\begin{array}{cc}
2 P(t)-1 & 2 P(t)^{2}-2 P(t) \\
2 & 2 P(t)-1
\end{array}\right)\left(\begin{array}{l}
U_{n} \\
V_{n}
\end{array}\right) \\
= & \left(\begin{array}{c}
(2 P(t)-1) U_{n}+\left(2 P(t)^{2}-2 P(t)\right) V_{n} \\
2 U_{n}+(2 P(t)-1) V_{n}
\end{array}\right)
\end{aligned}
$$

Hence, we conclude that

$$
\begin{aligned}
& U_{n+1}^{2}-\left(P(t)^{2}-P(t)\right) V_{n+1}^{2} \\
&= {\left[(2 P(t)-1) U_{n}\left(2 P(t)^{2}-2 P(t)\right) V_{n}\right]^{2} } \\
&-\left(P(t)^{2}-P(t)\right)\left[2 U_{n}+(2 P(t)-1) V_{n}\right]^{2} \\
&= U_{n}^{2}-\left(P(t)^{2}-P(t)\right) V_{n}^{2}=1
\end{aligned}
$$

So $\left(U_{n+1}, V_{n+1}\right)$ is also solution of $\tilde{E}$

3) Using (9), we find that

$$
\left\{\begin{array}{l}
U_{n}=(2 P(t)-1) U_{n-1}+\left(2 P(t)^{2}-2 P(t)\right) V_{n-1} \\
V_{n}=2 U_{n-1}+(2 P(t)-1) V_{n-1}
\end{array}\right.
$$

for $n \geq 2$

4) We prove it using the method of mathematical induction. For $n=4$, we get

$$
\begin{aligned}
& U_{1}=2 P(t)-1 \\
& U_{2}=8 P(t)^{2}-8 P(t)+1 \\
& U_{3}=32 P(t)^{2}-48 P(t)^{2}+18 P(t)-1
\end{aligned}
$$

and

$$
U_{4}=128 P(t)^{4}-256 P(t)^{3}+160 P(t)^{2}-32 P(t)+1
$$

Hence

$$
\begin{aligned}
U_{4} & =128 P(t)^{4}-256 P(t)^{3}+160 P(t)^{2}-32 P(t)+1 \\
& =(4 P(t)-3)\left[32 P(t)^{3}-40 P(t)^{2}+10 P(t)\right] \\
& -(2 P(t)-1) \\
& =(4 P(t)-3) \\
& \cdot\left[32 P(t)^{2}-48 P(t)^{2}+18 P(t)-1+8 P(t)^{2}-8 P(t)+1\right] \\
& -(2 P(t)-1) \\
& =(4 P(t)-3)\left(U_{3}+U_{2}\right)-U_{1}
\end{aligned}
$$

So $U_{n}=(4 P(t)-3)\left(U_{n-1}+U_{n-2}\right)-U_{n-3}$ is satisfied for $n=4$. Let us assume that this relation is satisfied for $n$, that is,

$$
U_{n}=(4 P(t)-3)\left(U_{n-1}+U_{n-2}\right)-U_{n-3}
$$

Then using (9) and (10), we conclude that

$$
U_{n+1}=(4 P(t)-3)\left(U_{n}+U_{n-1}\right)-U_{n-2}
$$

completing the proof.

Similarly, we prove that

$$
V_{n}=(4 t-3)\left(V_{n-1}+V_{n-2}\right)-V_{n-3}, \forall n \geq 4
$$

5) We prove it using the method of mathematical induction. For $n=1$, we have

$$
\begin{aligned}
\frac{U_{1}}{V_{1}} & =\frac{2 P(t)-1}{2}=\frac{2 P(t)-2+1}{2}=P(t)-1+\frac{1}{2} \\
& =[P(t)-1 ; 2]
\end{aligned}
$$

which is the fundamental solution of $\tilde{E}$. Let us assume that the $n$-th solution $\left(U_{n}, V_{n}\right)$ is given by

$$
\frac{U_{n}}{V_{n}}=[P(t)-1 ; \underbrace{2,2 P(t)-2, \cdots, 2,2 P(t)-2}_{n-1 \text { times }}, 2]
$$

and we show that it holds for $\left(U_{n+1}, y_{n+1}\right)$.

Using (6) , we have 


$$
\begin{aligned}
\frac{U_{n+1}}{V_{n+1}} & =\frac{(2 P(t)-1) U_{n}+\left(2 P(t)^{2}-2 P(t)\right) V_{n}}{2 U_{n}+(2 P(t)-1) V_{n}}=\frac{2(P(t)-1) U_{n}+U_{n}+(2 P(t)-1)(P(t)-1) V_{n}+(P(t)-1) V_{n}}{2 U_{n}+(2 P(t)-1) V_{n}} \\
& =P(t)-1+\frac{1}{2+\frac{1}{P(t)-1+\frac{U_{n}}{V_{n}}}}
\end{aligned}
$$

as

$$
\begin{aligned}
& P(t)-1+\frac{U_{n}}{V_{n}}=P(t)-1+P(t)-1 \\
& +\frac{1}{2+\frac{1}{\cdots+\frac{1}{2 P(t)-2+\frac{1}{2}}}} \\
& =2 P(t)-2 \\
& +\frac{1}{2+\frac{1}{2 P(t)-2+\frac{1}{\cdots+\frac{1}{2 P(t)-2+\frac{1}{2}}}}}
\end{aligned}
$$

we get

$$
\begin{aligned}
& \frac{U_{n+1}}{V_{n+1}}=P(t)-1 \\
& +\frac{1}{2+\frac{1}{2 P(t)-2+\frac{1}{2+\frac{1}{2 P(t)-2+\frac{1}{\cdots+\frac{1}{2 P(t)-2+\frac{1}{2}}}}}}} \\
& =[P(t)-1 ; \underbrace{2,2 P(t)-2, \cdots, 2,2 P(t)-2}_{n \text { times }}, 2]
\end{aligned}
$$

completing the proof.

As we reported above, the Diophantine equation $E$ could be transformed into the Diophantine equation $\tilde{E}$ via the transformation $T$. Also, we showed that $X=U+2 P(t)-1$ and $Y=V+2$. So, we can retransfer all results from $\tilde{E}$ to $E$ by applying the inverse of $T$. Thus, we can give the following main theorem:

Theorem 2.2: Let $D$ be the Diophantine equation in (1). Then

1) The fundamental (minimal) solution of $E$ is

$$
\left(X_{1}, Y_{1}\right)=(4 P(t)-2,4)
$$

2) Define the sequence

$$
\left\{\left(X_{n}, Y_{n}\right)\right\}_{n \geq 1}=\left\{\left(U_{n}+2 P(t)-1, V_{n}+2\right)\right\}
$$

where $\left\{\left(X_{n}, Y_{n}\right)\right\}$ defined in (5). Then $\left(X_{n}, Y_{n}\right)$ is a solution of $E$. So it has infinitely many integer solutions $\left(X_{n}, Y_{n}\right) \in \mathbb{Z} \times \mathbb{Z}$.

3) The solutions $\left(X_{n}, Y_{n}\right)$ satisfy the recurrence relations (see (11))

4) The solutions $\left(X_{n}, Y_{n}\right)$ satisfy the recurrence relations (see (12))

Example 2.3: Let $P(t)=2 t+1$, Then $\left(U_{1}, V_{1}\right)=(4 t+1,2)$ is the fundamental solution of

$$
\tilde{E}: U^{2}-\left(4 t^{2}+2 t\right) V^{2}=1
$$

and some other solutions are

$$
\begin{aligned}
& \left(\begin{array}{l}
U_{2} \\
V_{2}
\end{array}\right)=\left(\begin{array}{cc}
4 t+1 & 8 t^{2}+4 t \\
2 & 4 t+1
\end{array}\right)\left(\begin{array}{l}
4 t+1 \\
2
\end{array}\right)=\left(\begin{array}{l}
32 t^{2}+16 t+1 \\
128 t^{2}+64 t+5
\end{array}\right) \\
& \left(\begin{array}{l}
U_{3} \\
V_{3}
\end{array}\right)=\left(\begin{array}{cc}
4 t+1 & 8 t^{2}+4 t \\
2 & 4 t+1
\end{array}\right)\left(\begin{array}{l}
4 t+1 \\
2
\end{array}\right)=\left(\begin{array}{l}
256 t^{3}+192 t^{2}+36 t+1 \\
128 t^{2}+64 t+5
\end{array}\right)
\end{aligned}
$$

$$
\begin{aligned}
& \begin{cases}X_{n}=(2 P(t)-1) X_{n-1}+\left(2 P(t)^{2}-2 P(t)\right) Y_{n-1}-8 P(t)^{2}+10 P(t)-2 \\
Y_{n}=2 X_{n-1}+(2 P(t)-1) Y_{n-1}-8 P(t)+6 & \text { for } n \geq 2\end{cases} \\
& \begin{cases}X_{n}=(4 P(t)-3)\left(X_{n-1}+X_{n-2}\right)-X_{n-3}-16 P(t)^{2}+24 P(t)-8 \\
Y_{n}=(4 P(t)-3)\left(Y_{n-1}+Y_{n-2}\right)-Y_{n-3}-16 P(t)+16 & \text { for } n \geq 4\end{cases}
\end{aligned}
$$




$$
\begin{aligned}
\left(\begin{array}{l}
U_{4} \\
V_{4}
\end{array}\right) & =\left(\begin{array}{cc}
4 t+1 & 8 t^{2}+4 t \\
2 & 4 t+1
\end{array}\right)\left(\begin{array}{l}
4 t+1 \\
2
\end{array}\right) \\
& =\left(\begin{array}{l}
2048 t^{4}+2048 t^{3}+632 t^{2}+60 t+1 \\
1024 t^{3}+768 t^{2}+156 t+7
\end{array}\right) \\
\left(\begin{array}{l}
U_{5} \\
V_{5}
\end{array}\right) & =\left(\begin{array}{cc}
4 t+1 & 8 t^{2}+4 t \\
2 & 4 t+1
\end{array}\right)\left(\begin{array}{l}
4 t+1 \\
2
\end{array}\right) \\
& =\left(\begin{array}{c}
16192 t^{5}+20480 t^{4}+8896 t^{3}+1552 t^{2}+92 t+1 \\
8096 t^{4}+8192 t^{3}+2656 t^{2}+304 t+9
\end{array}\right)
\end{aligned}
$$

Further

$$
\begin{aligned}
\frac{U_{1}}{V_{1}} & =[2 t ; 2]=\frac{4 t+1}{2} \\
\frac{U_{2}}{V_{2}} & =[2 t ; 2,4 t, 2]=\frac{32 t^{2}+16 t+1}{16 t+4} \\
\frac{U_{3}}{V_{3}} & =[2 t ; 2,4 t, 2,4 t, 2]=\frac{256 t^{3}+192 t^{2}+36 t+1}{128 t^{2}+64 t+5} \\
\frac{U_{4}}{V_{4}} & =[2 t ; 2,4 t, 2,4 t, 2,4 t, 2] \\
& =\frac{2048 t^{4}+2048 t^{3}+632 t^{2}+60 t+1}{1024 t^{3}+768 t^{2}+156 t+7} \\
\frac{U_{5}}{U_{5}} & =[2 t ; 2,4 t, 2,4 t, 2,4 t, 2,4 t, 2] \\
& =\frac{16192 t^{5}+20480 t^{4}+8896 t^{3}+1552 t^{2}+92 t+1}{8096 t^{4}+8192 t^{3}+2656 t^{2}+304 t+9}
\end{aligned}
$$

It can be concluded now, that the fundamental solution of

$$
E: X^{2}-\left(4 t^{2}+2 t\right) Y^{2}-(4 t+2) X+\left(16 t^{2}+8 t\right) Y=0
$$

is $(8 t+2,4)$. Some other solutions are

$$
\begin{aligned}
& \left(\begin{array}{l}
X_{2} \\
Y_{2}
\end{array}\right)=\left(\begin{array}{c}
32 t^{2}+20 t+2 \\
16 t+6
\end{array}\right) \\
& \left(\begin{array}{l}
X_{3} \\
Y_{3}
\end{array}\right)=\left(\begin{array}{c}
256 t^{3}+192 t^{2}+40 t+2 \\
128 t^{2}+64 t+7
\end{array}\right) \\
& \left(\begin{array}{l}
X_{4} \\
Y_{4}
\end{array}\right)=\left(\begin{array}{c}
2048 t^{4}+2048 t^{3}+632 t^{2}+64 t+2 \\
1024 t^{3}+768 t^{2}+156 t+9
\end{array}\right) \\
& \left(\begin{array}{l}
X_{5} \\
X_{5}
\end{array}\right)=\left(\begin{array}{c}
16192 t^{5}+20480 t^{4}+8896 t^{3}+1552 t^{2}+96 t+2 \\
8096 t^{4}+8192 t^{3}+2656 t^{2}+304 t+11
\end{array}\right)
\end{aligned}
$$

\section{Acknowledgements}

We would like to thank Saäd Chandoul and Massöuda Loörayed for helpful discussions and many remarks.

\section{References}

[1] I. Niven, H. S. Zuckerman and H. L. Montgomery, “An Introduction to the Theory of Numbers," 5th Edition, Oxford University Press, Oxford, 1991.

[2] A. Tekcan, “The Pell Equation $x^{2}-D y^{2}= \pm 4$," Applied Mathematical Sciences, Vol. 1, No. 8, 2007, pp. 363-369.

[3] P. Kaplan and K. S Williams, "Pell's Equation $x^{2}-m y^{2}=-1,-4$ and Continued Fractions,” Journal of Number Theory, Vol. 23, No. 2, 1986, pp. 169-182. doi:10.1016/0022-314X(86)90087-9

[4] K. Matthews, "The Diophantine Equation $x^{2}-D y^{2}=N, D>0$," Expositiones Mathematicae, Vol. 18, 2000, pp. 323-331.

[5] R. A. Mollin, A. J Poorten and H. C. Williams, "Halfway to a Solution of $x^{2}-D y^{2}=-3$," Journal de Theorie des Nombres Bordeaux, Vol. 6, No. 2, 1994, pp. 421-457.

[6] P. Stevenhagen, "A Density Conjecture for the Negative Pell Equation, Computational Algebra and Number Theory,” Math. Appl., Vol. 325, 1992, pp. 187-200.

[7] A. Chandoul, "The Pell Equation $x^{2}-D y^{2}= \pm 9$," $R e$ search Journal of Pure Algebra, Vol. 1, No. 2, 2011, pp. 11-15.

[8] A. S. Shabani, "The Proof of Two Conjectures Related to Pell's Equation $x^{2}-D y^{2}= \pm 4$," International Journal of Computational and Mathematical Sciences, Vol. 2, No. 1, 2008, pp. 24-27.

[9] A. Chandoul, "The Pell Equation $x^{2}-D y^{2}= \pm k^{2}$," Advances in Pure Mathematics, Vol. 1, No. 2, 2011, pp. 16-22. doi:10.4236/apm.2011.12005

[10] A. Dubickas and J. Steuding, "The Polynomial Pell Equation,” Elemente der Mathematik, Vol. 59, No. 4, 2004, pp. 133-143. doi:10.1007/s00017-004-0214-7

[11] A. Tekcan, "Quadratic Diophantine Equation $x^{2}-\left(t^{2}-t\right) y^{2}-(4 t-2) x+\left(4 t^{2}-4 t\right) y=0$, Bulletin of Malaysian Mathematical Society, Vol. 33, No. 2, 2010, pp. 273-280.

[12] A. Chandoul, "On Quadratic Diophantine Equation $x^{2}-\left(t^{2}-t\right) y^{2}-(16 t-4) x+916 t^{2}-16 t y=0,, \quad$ International Mathematical Forum, Vol. 6, No. 36, 2011, pp. 1777-1782. 